Des migrants environnementaux aux migrants climatiques : un enjeu définitionnel complexe

Chloé Anne Vlassopoulos

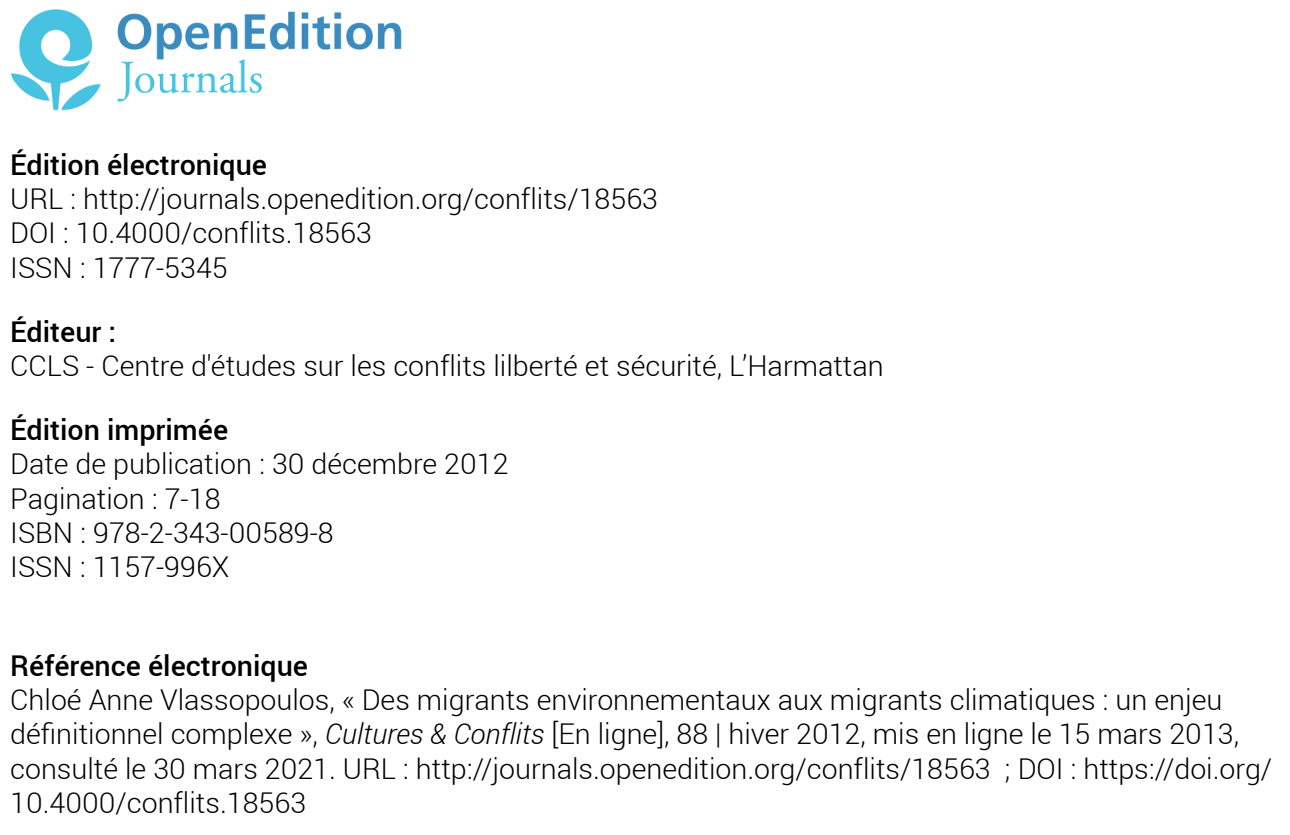

Creative Commons License 


\section{Des migrants environnementaux aux migrants climatiques : un enjeu définitionnel complexe}

\section{Chloé Anne VLASSOPOULOS}

Chloé Vlassopoulos est docteur en science politique de l'Université Paris II Panthéon-Assas et Maître de Conférences à l'Université de Picardie Jules Verne, spécialiste des politiques publiques de l'environnement. Elle est chercheur an Centre Universitaire de Recherche sur l'Action Publique et Politique (CURAPP/CNRS) et membre du comité de direction du réseau scientifique $T E R R A$ où elle dirige l'axe de recherche "Crises environnementales, changement climatique et migrations». Ses travaux portent plus particulièrement sur l'bistoire et la comparaison des politiques publiques, en relation avec la pollution de l'air et le changement climatique. Récemment elle s'est orientée sur les questions des migrations environnementales.

R éfugiés environnementaux/climatiques, migrants forcés de l'environne$\mathbf{R}_{\text {ment/du climat, migrants environnementaux/climatiques, éco- }}$ migrants... Les termes pour qualifier les personnes forcées de quitter leur lieu habituel de vie en raison d'une dégradation de l'environnement ne manquent pas et reflètent la difficile construction de cet enjeu en tant qu'objet de recherche et problème nécessitant l'intervention des autorités publiques.

L'histoire de l'humanité peut être représentée comme un processus continu de déplacements et d'adaptations à des conditions environnementales toujours changeantes. En ce sens, on pourrait soutenir a priori que nous sommes aujourd'hui face à une situation "normale » et non pas face à un "problème » nécessitant l'élaboration de mesures $a d$ hoc à différentes échelles territoriales. L'article de S. Bronkhorst dans ce numéro montre comment des populations nomades au Soudan ont appris progressivement à s'adapter aux conditions climatiques changeantes et comment la survenue d'interventions de régulation extérieures peut perturber les équilibres fragiles entre l'homme et la nature. Cependant, depuis les années 1980, le débat s'intensifie, surtout 
au niveau international, en vue de la reconnaissance et de la définition des migrations environnementales comme problème public.

Tout au long de ce débat trentenaire, plusieurs types d'acteurs (scientifiques, institutionnels, politiques...) se sont mis à interagir, sans forcément utiliser les mêmes termes pour qualifier le problème, sans même se référer au même problème. Pour certains, la migration environnementale (et/ou climatique) apparaît comme un problème autonome nécessitant la mise en place de politiques ad hoc. Pour d'autres, elle apparaît comme une conséquence de la dégradation environnementale, notamment du changement climatique, qui doit être abordée par des politiques environnementales plus rigoureuses. Pour d'autres encore, elle est perçue comme une solution au réchauffement de la planète qui doit être encouragée et organisée par le haut (top-down). Enfin, elle est aussi débattue comme une conséquence des politiques élaborées pour faire face à une crise environnementale ou climatique. Les articles proposés dans ce numéro font apparaître les différentes facettes du débat, révélant ainsi la complexité du lien entre migrations et environnement.

Sans prétendre reconstituer l'histoire détaillée de ce processus discursif, cette introduction propose une présentation du contexte dans lequel ce lien prend forme. Nous insisterons plus spécifiquement sur trois éléments qui permettent à la fois de comprendre les principaux points de friction entre acteurs et les glissements sémantiques opérés durant l'histoire de la construction du problème. Bien sûr, les acteurs et les perceptions dominantes sont interconnectés dans la mesure où les premiers sont porteurs de scénarios définitionnels qui en retour influent sur les rapports en présence. Il s'agit dans un premier temps de voir les conceptualisations concurrentes au sein de la communauté scientifique (1) qui offrent un premier cadrage pour l'appropriation politicoinstitutionnelle de cet enjeu (2). Il s'agit enfin d'aborder l'intégration de la question migratoire dans l'agenda climatique et ses implications en termes de définition des migrations environnementales comme problème public (3).

\section{Y a-t-il problème ? La communauté scientifique divisée}

La mise en liaison des migrations et de l'environnement n'est pas un fait révélé par son évidence objective, ni une invention des institutions internationales dans leur effort de s'attribuer un nouvel espace d'action. Cette mise en liaison s'est, dans un premier temps, opérée au sein de différentes communautés scientifiques, chacune offrant des rationalisations concurrentes de la réalité et aboutissant à des argumentations sur lesquelles s'appuieront, dans un deuxième temps, les acteurs institutionnels pour justifier leurs actions, ou non actions ${ }^{1}$.

1. Gusfield J., The culture of public problems. Drinking, driving and the symbolic order, Chicago, The University of Chicago Press, 1984. 
En effet, depuis l'émergence des premiers travaux sur les « réfugiés » de l'environnement, la communauté scientifique s'est trouvée clivée entre ceux qui annoncent une catastrophe imminente, celle de mouvements migratoires de masse liés à une dégradation sévère de l'environnement naturel (maximalistes) et ceux qui ne voient dans l'environnement qu'un facteur parmi d'autres poussant les gens à fuir leur lieu habituel de vie (minimalistes) ${ }^{2}$. Ces deux groupes n'étant pas homogènes, nous pouvons distinguer en leur sein des perceptions divergentes de l'enjeu.

Les maximalistes sont caractérisés par un discours alarmiste qui consiste à avancer des chiffres portant sur des millions de personnes risquant de se voir condamnées à la migration, victimes d'un environnement devenu inhabitable. Les premiers à avoir développé une telle approche sont les spécialistes de l'environnement mais leur rapport au « nouveau » problème migratoire reste paradoxal. D'une part, ils ont servi de « lanceurs d'alerte » au sens où, dans un contexte d'incertitude, ils ont créé une nouvelle catégorie cognitive permettant de donner sens à un fait perçu comme pouvant constituer un danger pour l'homme et/ou son environnement ${ }^{3}$. D'autre part, plus que la construction d'un problème autonome, devant faire l'objet d'une politique ad hoc, l'objectif premier des spécialistes de l'environnement a été de mettre l'accent sur la gravité d'un autre problème, celui de la dégradation environnementale, et sur la nécessité de renforcer les mesures nécessaires pour y remédier.

E. El Hinnawi, universitaire égyptien, spécialiste des questions environnementales et énergétiques et consultant auprès du Centre International pour l'environnement et le développement, est le premier à avoir rédigé un rapport pour le PNUE en 1985 en médiatisant le terme " refugiés environnementaux ». En 1988, J. Jacobson, membre du WorldWatch Institute reprend le travail de Hinnawi pour rédiger un rapport soutenant l'existence d'un lien causal direct entre environnement et mouvements migratoires. Il estime le nombre de refugiés environnementaux à 10 millions en $1988^{4}$. N. Myers, professeur en sciences environnementales, multiplie ses publications depuis les années 1990 et participe à la politisation de l'enjeu par les relations qu'il entretient au sein des instances internationales. Il évoque la présence de 25 millions réfugiés environnementaux en 1997, et en annonce 200 millions pour 20505.

Cette mise sur l'agenda scientifique par les environnementalistes de l'enjeu « réfugiés environnementaux » dans les années 1980 ne doit pourtant pas

2. Suhrke A., "Environmental degradation and migration flows", Journal of International Affairs, 2-47, 1994, pp. 437-496.

3. Chateauraynaud F., Argumenter dans un champ de forces, essai de balistique sociologique, Paris, Petra, 2011.

4. Jacobson J.L., "Environmental Refugees: a Yardstick of Habitability", Worldwatch Paper, 86, Washington DC, Worldwatch Institute, 1988.

5. Myers N., “'Environmental Refugees”, Population and Environment, 2-19, 1997, pp. 167182. 
dissimuler toute une série de recherches effectuées, depuis les années 1970, dans le cadre d'études sur la sécurité qui n’ont pas eu la même visibilité, peutêtre parce que les questions environnementales s'exportent mieux dans les sphères non académiques et que l'agenda sécuritaire est resté longtemps dominé par les enjeux de la guerre froide. Ces recherches mettent l'accent sur le lien entre dégradation environnementale et conflit. Elles identifient le facteur migratoire comme étant à la fois une conséquence et une cause des crises environnementales elles-mêmes potentiellement génératrices de conflits. Ici, aussi, le but des chercheurs n'est pas de définir un nouveau problème mais de proposer une nouvelle lecture des enjeux sécuritaires à travers le prisme de la dégradation des ressources naturelles. Ainsi, en 1977, L. Brown appelle à une redéfinition de l'agenda traditionnel en matière de sécurité pour inclure les défis écologiques ${ }^{6}$. Il est d'ailleurs le premier à avoir utilisé le terme « environmental refugee » en $1976^{7}$ (voir dans ce numéro l'article de K. McNamara et C. Gibson). En 1978, D. Pirages utilise le terme « ecopolitics» comme une nouvelle approche des relations internationales et W. Ophuls insiste sur le déséquilibre entre ressources disponibles et pressions démographiques en adoptant le terme de rareté écologique ${ }^{8}$. Plus récemment, T. Homer-Dixon soutient que la dégradation et l'épuisement des ressources naturelles et l'accès inégal à ces ressources, composés avec les pressions démographiques, produisent ce que l'auteur qualifie de " rareté environnementale » 9 . La rareté environnementale, soutient Homer-Dixon, est une cause majeure de conflits entre États qui peuvent prendre la forme de conflits identitaires entre groupes ou de ruptures institutionnelles générant des guerres civiles (pour une approche critique de ces travaux voir l'article de J. Shade dans ce numéro). Inspirés de ces travaux, N. Myers et J. Kent rapprochent les deux communautés de recherche et les deux problématiques en qualifiant les refugiés environnementaux, non seulement comme un enjeu environnemental mais aussi comme un enjeu sécuritaire ${ }^{10}$.

Comme le remarque Suhrke, la communauté des « minimalistes » se compose essentiellement de chercheurs travaillant sur les questions migratoires. MM. Kritz, R.A. Bilsborrow, G. Hugo, G. Kibreab ou R. Black abordent tous ces questions et défendent la même thèse : la migration humaine est un problème complexe lié à une multitude de causes, dont celle environnementale ${ }^{11}$.

6. Brown L., "Redefining national security", Worldwatch Paper, 14, Washington DC, Worldwatch Institute, 1977.

7. Brown L., Mcgrath P., Stokes B., “Twenty two dimensions of the population problem”, Worldwatch Paper, 5, Washington DC, Worldwatch Institute, 1976.

8. Pirages D., Global Ecopolitics: The new context for international relations, North Scituate, MA: Duxbury Press, 1978 ; Ophuls W., Ecology and the politics of scarcity, San Fancisco, Freeman, 1977.

9. Homer-Dixon T.F., "Environmental Scarcities and Violent Conflict: Evidence from Cases”, International Security, 1-19, 1994, pp. 5-40.

10. Myers N., Kent J., "Environmental Exodus: An Emergent Crisis in the Global Arena”, The Climate Institute, Washington DC, 1995.

11. Kritz M.M., "Climate change and migration adaptations”, Working paper series, Cornell 
L'environnement ne doit donc pas être perçu comme un facteur autonome et suffisant à lui seul pour générer un processus de déplacement de populations. Ayant travaillé, pour beaucoup d'entre eux, sur les questions de l'asile politique, ils mettent également en garde contre l'utilisation du terme "réfugié » qui risquerait de fragiliser les conditions d'application de la Convention de Genève (1951), rencontrant déjà des problèmes d'applicabilité dans les pays occidentaux. Une distinction nous semble cependant nécessaire au sein de cette communauté scientifique car les juristes travaillant sur le droit international de la migration et de l'asile se sont souvent montrés plus favorables à une reconnaissance officielle des migrants environnementaux et à la mise en place d'instruments spécifiques de protection (voir M. Morel et N. de Moor dans ce numéro et V. Magniny 12). Se ralliant à l'initiative du Centre International de Droit Comparé de l'Environnement (CIDRE), ils ont appuyé l'Appel de Limoges qui revendique la reconnaissance d'un statut international pour les réfugiés écologiques ${ }^{13}$.

\section{Un problème ou des problèmes ? le malaise institutionnel}

Une communauté scientifique fragmentée, émettant des discours dissonants sur l'existence d'un nouveau problème dit de migrations environnementales et ceci dans un contexte politique marqué par la fermeture des frontières des pays occidentaux aux candidats à la migration, sont autant de facteurs qui freinent la définition de cet enjeu en tant que problème autonome d'action publique. Les politiques publiques procèdent toujours par simplification des problèmes qu'elles visent à réguler ${ }^{14}$ mais pour qu'il y ait élaboration d'une politique publique il faut de prime abord que les problèmes à traiter aient fait l'objet d'une définition claire, ne serait ce qu'éphémère, et qu'ils soient perçus comme politiquement et administrativement « gérables »15. Les institutions n'aiment pas la complexité et l'imprécision. Mis à part le contexte politique défavorable, force est de constater dans le cas qui nous intéresse dans ce numéro, qu'il n'y a pas un consensus clair entre experts sur la question de

University, 1990 ; Bilsborrow R.W., "Rural poverty, migration and the environment in developing countries: three case studies”, article pour le Rapport sur le développement dans le monde : Le défi du développement, Banque Mondiale, WDR, 1991 ; Homer-Dixon T.F., op.cit. ; Hugo G., "Environmental Concerns and International Migration”, International Migration Review, 1-30, 1996, pp.105-131 ; Kibreab G., "Environmental Causes and Impact of Refugee Movements: A Critique of the Current Debate”, Disasters, 1-21, 1997, pp. 20-38 ; Black R., "Environmental Refugees: Myth or Reality?”, New Issues in Refugee Research, Working Paper, 34, Geneva, UNHCR, 2001.

12. Magniny V., Les réfugiés de l'environnement, hypothèse juridique à propos d'une menace écologique, Thèse de droit, Université de Paris Panthéon Sorbonne, 1999.

13. Cournil C., «A la recherche d'une protection pour les "réfugiés environnementaux" : actions, obstacles, enjeux et propositions ", Revue Asylon(s), n' 6, novembre, 2008, http://www.reseau-terra.eu/rubrique154.html.

14. Stone D., Policy Paradox. The art of political decision-making, N.Y., Norton, 1997.

15. Vlassopoulos C.A., "Defining environmental migration in the climate change era. Problem, consequence or solution?", in Faist T., Shade J., Disentangling Migration and Climate Change: Toward an analysis of Concepts, Methodologies, and Policies, Springer, 2012 (à paraître). 
savoir s'il y a problème ou pas et en quoi il consiste précisément. De surcroît, les définitions proposées par les défenseurs de la thèse alarmiste restent particulièrement floues en agrégeant des phénomènes difficilement compatibles sur le plan institutionnel.

Si l'on se réfère par exemple aux définitions proposées par El-Hinnawi et Myers, l'imprécision dans l'indentification de celui qui doit être perçu comme « refugié » de l'environnement devient tout de suite apparente. El-Hinnawi définit ces réfugiés comme « des personnes forcées de quitter leur habitation traditionnelle et permanente en raison d'une rupture environnementale significative (naturelle ou produite par l'homme) qui met en danger leur existence et affecte leur qualité de vie ${ }^{16}$ ». Quant à N. Myers, il qualifie les réfugiés environnementaux comme « des personnes ne pouvant plus avoir une vie sûre sur leurs lieux d'habitation quotidiens en raison de sécheresses, érosion du sol, désertification et autres problèmes environnementaux 17 ». La définition d'un problème sur l'agenda politique nécessite de préciser à la fois ses causes et ses conséquences. La définition des causes précise qui est (sont) responsable(s) et doi(ven)t assumer le coût de sa résolution. La définition des conséquences permet de justifier pourquoi une situation doit être perçue comme problématique et faire l'objet d'une politique publique. C'est cette double définition qui oriente le partage du pouvoir entre autorités chargées de traiter le problème. Or, l'imprécision qui règne en la matière fait apparaître plusieurs scénarios définitionnels et plusieurs autorités potentiellement compétentes.

Dans une tentative de clarification du débat, S. Lonergan établit une classification des causes potentielles de déplacement, montrant par-là la pluralité des situations que ces définitions recouvrent : l'épuisement des ressources naturelles (sol, eau, arbres), les accidents industriels, les catastrophes naturelles, les projets d'aménagement, les dommages écologiques militaires. Un regard plus attentif sur ces causes fait apparaître que différents secteurs d'action publique ont compétence pour adopter des mesures en vue de leur atténuation ${ }^{18}$. Pour ce qui concerne la gestion des ressources naturelles et la lutte contre les pollutions, le secteur de l'environnement élabore à la fois des politiques préventives en application des principes de prévention et de précaution et des politiques curatives, en application du principe pollueur-payeur, afin de limiter la dégradation de l'environnement, rétablir les équilibres écologiques et dédommager les victimes. Dans les cas de catastrophes naturelles, de travaux d'aménagement ou de conflits armés, le secteur humanitaire est le plus à même d'élaborer des mesures d'aide et d'assistance aux sinistrés et déplacés.

16. El-Hinnawi E., Environmental Refugees, UNEP, Nairobi, 1985.

17. Myers N., "Environmental Refugees in a globally warmed world", Bioscience, 11-43, 1993, pp.752-761.

18. Même si, dans son ouvrage de 1995, Myers propose certaines catégorisations, il continue de considérer le terme «environmental refugees » comme pouvant englober tout type de déplacement environnemental donnant ainsi à tort l'impression de la présence d'un problème « unifié » (voir K. McNamara et C. Gibson, infra). 
Lorsque les personnes déplacées se voient obligées de franchir les frontières nationales, le secteur de l'asile a compétence pour les assister. Ce dernier peut également apparaître compétent en cas de submersion d'archipels suite à la montée des eaux de mer, risquant de transformer les populations indigènes en « apatrides ${ }^{19}$.

Tout une panoplie de textes internationaux existent d'ailleurs qui, sans explicitement évoquer la question migration-environnement, proposent des instruments juridiques prenant en considération, de manière plus ou moins explicite, la question des populations touchées (voir l'analyse de Morel et $\mathrm{N}$. de Moor). À titre d'exemple, nous pouvons citer la Convention sur la lutte contre la désertification qui reconnaît la liaison entre dégradation environnementale et migrations en parlant de « la corrélation qui existe entre les phénomènes de désertification, de sécheresse $[. .$.$] et ceux qui découlent des migra-$ tions, des déplacements de populations et de la dynamique démographique » (Préambule). La Convention sur la biodiversité reconnaît quant à elle «la dépendance des communautés indigènes locales et de leur mode de vie traditionnel, au maintien des ressources biologiques 20 ». La Convention sur les effets transfrontaliers des accidents industriels reconnait explicitement la responsabilité des exploitants-pollueurs. La Convention de Genève et la Convention sur l'apatridie peuvent aussi servir de cadre pour gérer les souffrances générées par certaines crises climatiques ${ }^{21}$.

Force est de constater que derrière le terme, en apparence unificateur, de « réfugié/migrant environnemental » se profilent différents problèmes (environnementaux, humanitaires, asilaires,...) qui se font concurrence lors du processus de mise sur agenda. Chaque secteur, en fonction de son référentiel et de ses compétences, perçoit des enjeux différents qu'il tente de mettre en avant, renforçant ainsi sa légitimité et son autorité vis-à vis des autres. Le malaise des institutions quant à savoir si quelqu'un (et qui) doit devenir le « porteur » d'un problème dit des migrants environnementaux ou climatiques devient visible par les réticences et tergiversations des agences internationales qui avancent en la matière de manière incrémentale (voir l'article de $\mathrm{K}$. McNamara et $\mathrm{C}$. Gibson). Il devient aussi visible par la rivalité qui caractérise certaines d'entre elles pour l'imposition d'une définition compatible avec leurs attributions ${ }^{22}$.

19. Vlassopoulos C. A., "Institutional barriers to the recognition and assistance of environmentally forced migrants” in Afifi T., Jäger J. (eds), Environment, forced migration and vulnerability, Springer-Verlag, Berlin and Heidelberg GmbH \& Co, 2010.

20. Convention internationale sur la lutte contre la désertification dans les pays gravement touchés par la sécheresse et/ou la désertification. http://www.unccd.int/Lists/SiteDocumentLibrary/conventionText/conv-fre.pdf

21. L'article signé, en 2008, par le Haut Commissaire pour les réfugiés à l'ONU va dans ce sens lorsqu'il reconnaît que certains mouvements induits par le changement climatique peuvent en effet relever du cadre de la Convention de 1951 sur les réfugiés. Par ailleurs, le rapport du comité exécutif du programme du Haut Commissaire, en 2006, reconnaissant que le dispositif sur l'apatridie est sous-utilisé, incite à une meilleure mobilisation pour l'identification, la prévention et la réduction des cas d'apatridie et la protection des apatrides.

22. Vlassopoulos C. A., 2012, op.cit. 


\section{La dynamique climatique comme fenêtre d'opportunité}

Le débat public, qui se construit progressivement sur l'arène internationale, étant soutenu par une production scientifique de plus en plus abondante, marque un tournant dans les années 2000 lorsque l'enjeu des migrations environnementales rencontre celui du changement climatique. Le Groupe d'Experts Intergouvernemental sur l'Évolution du Climat (GIEC), dans son rapport de 1990, se référait déjà au fait qu' " un des impacts les plus importants $\mathrm{du}$ changement climatique peut être celui sur la migration humaine 23 ». Cependant, cette affirmation ne s'est pas avérée suffisante pour attirer l'attention sur la question des migrations climatiques. C'est surtout le succès qu'a connu le changement climatique, imposé dans les années 2000 comme un problème majeur dans l'arène internationale, qui a servi d'aimant attirant autour de lui différents enjeux qui, depuis, sont débattus dans une perspective climatique. La question migratoire en est un exemple.

Comme le montre J-F Mouhot dans son article, l'interaction homme-climat constitue une préoccupation philosophique, scientifique et politique qui remonte très loin dans le temps (présente déjà dans l'épopée de Gilgamesh au XVIII-XVIe siècle avant J.C.). Ayant traversé les siècles, cette question a donné lieu à diverses controverses dont celle des années récentes qui ne doit être perçue que comme un fragment du processus définitionnel d'un problème en perpétuelle construction et reconstruction. Ne serait-ce que depuis les années 1980, le changement climatique fait l'objet de différentes conceptualisations. D'abord perçu comme un problème environnemental et débattu au sein de communautés spécialisées en cette matière (PNUE, WMO, GIEC,...), il connaît, dans les années 2000, une forte complexification. Il ne s'agit plus seulement de chercher à identifier et atténuer ses causes (diverses sources de gaz à effet de serre). Il s'agit aussi de s'intéresser et gérer ses conséquences sur l'homme (pauvreté, sécurité, migration...) ${ }^{24}$. La notion de « développement durable », imposée comme un objectif à atteindre sur l'échelle internationale, va servir de « liant » permettant la mise en parallèle (et non la fusion) des deux approches du problème climatique, l'une écocentrique et l'autre anthropocentrique.

Depuis le sommet mondial sur le développement durable, le débat a essentiellement porté sur la question de savoir comment garantir un développement durable et non plus comment protéger l'environnement en soi ${ }^{25}$. En effet,

23. IPCC First Assesment Report, Working group III. http://www.ipcc.ch/publications_and_data/publications_and_data_reports.shtml\#.UP_psG $\mathrm{dw} 7 \mathrm{~h} 4$

24. Vlassopoulos C. A., "Competing definition of climate change and the post-Kyoto negotiations", International Journal in Climate Change Strategies and Management, 1-4, 2012, pp. 104-119.

25. Mee L., "The Role of UNEP and UNDP in Multilateral Environmental Agreements", International Environmental Agreements, 5, 2005, pp.227-263. 
cette évolution a permis à différentes institutions internationales et $\mathrm{ONG}$, non environnementales, d'entrer dans l'espace du débat climatique, ce qui leur offrait de nouvelles opportunités d'action, de la reconnaissance et de nouvelles sources de financement. Le souci de ces nouveaux acteurs n'est plus de savoir comment atténuer les émissions atmosphériques mais comment adapter les communautés humaines au réchauffement de la planète de façon à ne pas aggraver les situations de sous-développement, à faire face au risque de déplacement de populations et à prévenir l'émergence de conflits. Les chercheurs en sciences sociales se sont également adaptés à ces évolutions pour produire de nouvelles recherches sur les risques climatiques qui ont alimenté les récents rapports du GIEC.

En quoi la mise en avant des enjeux migratoires du changement climatique a-t-elle permis de faire évoluer un débat politico-scientifique long de vingt ans ? Tout d'abord, cette évolution définitionnelle a permis la simplification du problème. En effet, les différentes causes environnementales génératrices de migrations commencent à reculer dans les discussions qui se voient de plus en plus concentrées sur la seule cause climatique. Dans ce contexte, toutes les autres catégories de personnes déplacées suite, par exemple, à la construction de barrages, aux accidents industriels, aux destructions stratégiques de l'environnement se transforment, de fait, en non-problèmes et se voient effacées de l'agenda international. Cette simplification va permettre de rendre le problème plus gérable, ce qui aboutira en effet, pour la première fois, à un accord écrit entre les membres du Groupe de travail spécial sur l'action concertée à long terme au titre de la Convention sur le climat. Cet accord invite les parties à « entreprendre des actions pour améliorer la compréhension et la coopération [...] sur les questions de déplacements, de migration et, le cas échéant, de réinstallation planifiée liées au changement climatique $26 »$.

Ensuite, la concentration du discours sur les migrations climatiques va produire un glissement dans la mesure où les migrants/réfugiés environnementaux n'apparaissent plus sur l'agenda international comme un problème « autonome » mais plutôt comme une des conséquences d'un autre problème, celui du changement climatique. Il s'agit dans ce cas de gérer la question migratoire non pas par l'adoption de politiques publiques ad boc mais par des mesures dont l'objectif principal affiché est celui de lutter contre le changement climatique. Cette situation comporte une double limite. Dans un premier temps, derrière la discussion générale sur le problème global du climat qui change, le besoin d'identifier les responsables à l'origine de la souffrance des personnes déplacées se voit effacé (sur ce point voir aussi l'article de K. McNamara et C. Gibson), ce qui évacue du coup la question de la compensation des victimes et leur protection qui n'est plus au cœur de la problématique d'intervention. Dans ce contexte, la promotion par l'OIM d'une politique de 
migrations planifiées (de travail), pour les régions connaissant une crise climatique sévère et prolongée, est présentée comme une stratégie "gagnantgagnant » : profitable pour les gens, ayant perdu leurs moyens de subsistance suite à la crise climatique, qui trouvent du travail ailleurs et sont en mesure d'envoyer des fonds chez eux pour soutenir les membres de la famille restés au pays ; profitable aussi pour l'environnement étant donné que les ressources naturelles sont moins sollicitées par la diminution de la population sur le territoire concerné. Ce scénario d'action publique écarte, en effet, tout effort d'attribution de responsabilités et surtout ne questionne pas le ressentiment des gens qui, après avoir perdu leurs moyens de subsistance, se voient proposer de quitter leur pays et leur famille et de se transformer en travailleurs bon marché (cette nouvelle orientation politique est diversement commentée au sein de ce numéro).

Dans un deuxième temps, l'intégration de la question migratoire comme composante des politiques climatiques donne la fausse impression de compatibilité entre deux objectifs pas forcément concordants. Lutter contre le changement climatique est perçu comme n'ayant que des effets positifs sur l'enjeu migratoire. Cette perception simplifie à outrance des phénomènes particulièrement complexes. En ce sens, J. Schade (dans ce numéro) montre comment des politiques adoptées au nom du changement climatique peuvent devenir elles-mêmes sources de migrations.

Ce numéro spécial commence par un article de J-F Mouhot, qui aborde la relation homme-climat dans une dimension historique. Il montre en particulier que la question climatique et ses effets sur l'homme et l'impact de l'homme sur le climat ont toujours fait l'objet de débats dont le contenu a changé au fil du temps et en fonction des préoccupations et des connaissances scientifiques du moment. Dans un premier temps, l'auteur présente les grandes étapes de la prise de conscience du risque climatique et retrace la manière dont les hommes ont appréhendé le rapport au temps qu'il fait et à ses variations au cours des siècles. Dans un deuxième temps, il expose les domaines d'investigation de l'histoire en matière de changement climatique et s'interroge sur la place qu'occupent les historiens dans les débats sur le climat.

K. McNamara et C. Gibson effectuent une analyse de documents publiés ces 40 dernières années par les Nations Unies sur la question de la mobilité humaine due aux changements environnementaux. Alors que l'ONU joue un rôle majeur dans la protection de l'environnement et des personnes en déplacement forcé, le rapprochement de ces deux objectifs sous une même problématique semble avoir échoué. En identifiant les différentes catégorisations faites de ces personnes, les auteurs constatent une altération progressive des termes du débat qui n'aborde ni la question de l'attribution des responsabilités quant à la dégradation de l'environnement dans les régions touchées par 
des phénomènes migratoires, ni la question de la construction d'un cadre légal pour la protection des migrants environnementaux.

M. Morel et N. De Moor recensent les instruments juridiques offerts par quatre branches du droit international (droit international de l'environnement, droit international des droits de l'Homme, droit international de l'asile et droit international des migrations) qui peuvent servir à la protection des migrants climatiques. Ils soutiennent que, vue séparément, chacune de ces branches du droit joue un rôle spécifique et important mais néanmoins limité dans la gestion des migrations climatique. En revanche, considérées comme un ensemble, elles offrent un cadre de protection satisfaisant. Ils soutiennent, par ailleurs, que le droit des migrations peut se révéler aujourd'hui particulièrement pertinent pour les migrants climatiques ayant quitté leur pays pour devenir migrants du travail dans un pays étranger.

J. Schade, dans son article, observe que le discours sur les migrations climatiques est concentré sur la question des impacts du changement climatique en matière migratoire, ignorant le fait que les politiques climatiques d'atténuation et/ou d'adaptation peuvent être, elles-mêmes, source de déplacements des populations et de fragilisation de communautés déjà vulnérables. Elle appuie sa thèse sur une série d'exemples et soutient que l'absence de réflexion, à ce jour, sur le rapport entre politiques climatiques et migrations est liée à l'emprise des approches néo-malthusiennes au sein des communautés scientifiques et politiques travaillant sur le changement climatique et les enjeux migratoires. Elle met en garde contre la montée en puissance récente des plans de migrations planifiés (du travail) et opte pour le renforcement du respect des droits de l'Homme au sein de la Convention-cadre des Nations Unies sur les Changements Climatiques afin de renforcer les populations locales contre les risques induits par le régime climatique actuel.

A travers un cadre théorique qui lie le changement climatique, les migrations et l'émergence de conflits, S. Bronkhorst examine dans quelle mesure le pastoralisme peut être une forme d'adaptation au changement climatique dans l'État du Sud Kordofan. Elle constate que les conflits entre pasteurs et agriculteurs sont exacerbés suite à des interventions gouvernementales qui non seulement aggravent la rareté des ressources mais aussi affaiblissent les institutions locales ayant une longue expérience dans la gestion des ressources et des incompatibilités qui existent entre pratiques pastorales et agricoles. Dans ce contexte, alors qu'il s'agit d'un territoire rendu de plus en plus vulnérable par le changement climatique, la promotion du pastoralisme comme stratégie d'adaptation semble échouer.

Notre objectif dans ce numéro a été d'ouvrir le débat sur les migrations environnementales/climatiques en invitant des chercheurs travaillant en 
langue anglaise ${ }^{27}$. Nous espérons que cette invitation participera à l'enrichissement et au renforcement de la recherche effectuée en France sur ce sujet qui reste encore timide.

27. Nous souhaitons remercier V. Assadas, F. Jamay et J. Valluy pour la relecture attentive de la traduction des articles qui suivent. 